\title{
Research Article \\ Structure Dynamic Response of Amphibious Aircraft Induced by Water-Taxiing
}

\author{
Jihang Lyu $\mathbb{D}$, Rong Yang $(\mathbb{D}$, and Lingcai Huang $(\mathbb{D}$ \\ AVIC General Huanan Aircraft Industry Co. Ltd., 999 Jinhai Middle Road, Sanzao Town, Zhuhai City, Guangdong Province, China \\ 519040 \\ Correspondence should be addressed to Jihang Lyu; lvjihang@avicgeneral.com
}

Received 21 July 2021; Accepted 28 October 2021; Published 10 November 2021

Academic Editor: Giovanni Delibra

Copyright (C) 2021 Jihang Lyu et al. This is an open access article distributed under the Creative Commons Attribution License, which permits unrestricted use, distribution, and reproduction in any medium, provided the original work is properly cited.

\begin{abstract}
The significant dynamic response under the combined impact of aerodynamic and hydrodynamic forces could be likely to appear because of the structural flexibility, when taxiing on the water surface for amphibious aircraft. Meanwhile, the modal characteristics of the structure are also affected by the additional motion of water. These require that the influence of the structural elasticity and the coupling effect between water and structure should be considered in dynamic response analysis of water-taxiing. According to the peculiarities of the amphibious aircraft, structural dynamics model is based on the distribution of stiffness and mass, Virtual Mass Theory is utilized to solve the wet modes on the water surface, rational function approximations of unsteady aerodynamic force in time-domain are constructed by the Minimum-State Approximation Formula, and loose coupling method is employed to simulate the hydrodynamic elastic response under the encounter of amphibian with single wave and repeated waves, respectively. Analysis of dynamic characteristics during the water-taxiing of the amphibious aircraft has been achieved in this work. The results show that wet natural frequencies of the aircraft have different degrees of decline compared with the dry frequencies because of the influence of added water on the hull, and the response amplitude of dynamic loads obtained by using the wet modes have some certain extent decrease compared with the dry modes. The dynamic amplitude of different locations changes in different degree relatives to the center of gravity position, which reflects the influence of structural elasticity. Due to the excitation of single wave and repeated waves, the structural vibration amplitude will increase rapidly, but the amplitude shows a certain divergence trend under the action of repeated waves with a given oscillation frequency, which is more severe for structural strength design.
\end{abstract}

\section{Introduction}

Amphibious aircrafts possess the characteristics of both hydroplane and landplane and have the advantages of long endurance, strong environmental adaptability to take off and landing, superior flying performance at low altitude, and ultralow altitude. Therefore, they are widely used in fire fighting, maritime rescue, and other special missions. According to their own practical applications, amphibious aircrafts should have the abilities to repeatedly take off and land safely on the water surface during expected service life. However, amphibious aircrafts are often disturbed continuously by turbulence, waves, and so on during take off, landing, and taxiing on the water surface, which can easily cause the coupled vibrations of the aircraft structures, affecting the efficiency of the system equipments and the fatigue life of structures. Therefore, exploration of the dynamic characteristics of amphibious aircrafts can provide a significative basis for preventing structural resonance damage and vibrational fatigue and evaluating the structural dynamic characteristics and structural optimization design.

In the previous design of seaplanes and amphibious aircrafts, the water loads of different hull configurations and different aircraft weights are mainly calculated according to the empirical formula at take off and landing conditions, and all the loads obtained are static water loads; the structural dynamic action of hydrodynamic loads on rough water surface is not seriously considered $[1,2]$. The researches on aircraft hydrodynamics have been carried out mainly for the landing on waves or ditching stage, and the influence of 
structural elasticity has been universally ignored that the rigid models are used for numerical simulation [3-5]. Along with the higher speed and larger layout size of the amphibious aircraft, the combined action of aerodynamic and hydrodynamic forces will lead to the generation of structural dynamic response, which may become more significant under the effect of elasticity and has an impact on the structural safety.

The dynamic researches considering structural elasticity mainly focus on the airborne configuration of the aircraft presently [6] and including the seaplanes [7, 8]. Since the air density is much smaller than the material density of the aircraft structure, the influence of air on the structure modes can be ignored during the analysis. However, when a large amphibious aircraft is taxiing on the water surface, the movement of hull will induce the motion of surrounding water, and the water will react on the hull surface in the form of additional motion. Since the water density is much larger than the air density, which is equivalent to magnitude of material density of the aircraft structures, additional motion of the water will evidently affect structural dynamics of the hull $[9,10]$. Therefore, it is necessary to consider the effects of aerodynamic and hydrodynamic forces as well as the coupling influence of additional water motion on the structures and should employ the wet modes to analyze the dynamic response on the water surface of large amphibious aircraft.

Generally, for the multifield coupling dynamic problems such as water-taxiing of the aircraft, the coupling effect mainly occurs at the interface of the fields, so loose coupling or partitioned coupling can be adopted for appropriate simplification in engineering. The motion of the fluid filed and the structure are solved separately; the interaction between fluid and solid is realization through the load-bearing interface, so as to obtain a systemic physical explanation similar to the real situation.

Simultaneously, for the wet modal analysis of underwater structures, three-dimensional model of structures and surrounding water field should be established popularly. The water is popularly regarded as an inviscid, irrotational, and incompressible ideal fluid, and the influence of additional elastic force and damping force is usually ignored in the model. The more attention is paid to the effect of additional inertial force at the solid-liquid interface $[11,12]$. Specifically, the added mass of the structure underwater is calculated according to the coupling effect; the eigenvalues and eigenvectors are solved based on the new model with additional mass added to acquire the frequencies and mode shapes accordingly.

In this work, the structural wet modal model, aerodynamic model, and hydrodynamic load model of large amphibious aircraft are established individually. The Virtual Mass Theory is adopted to analyze the wet modes of large amphibious aircraft, the Minimum-State Approximation Formula is used for generalized aerodynamic time-domain fitting of lift surfaces, the hydrodynamic numerical simulation of elastic aircraft is carried out by loose coupling method, and the structural elastic responses are solved by mode superposition method. With that, the effect of the additional water motion on the structural modes and the

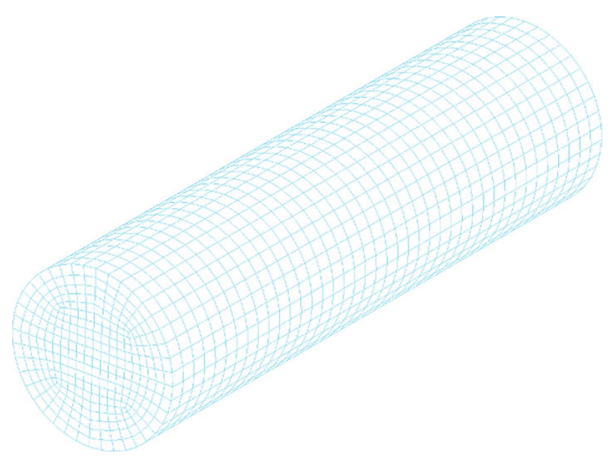

Figure 1: Finite element model of the cylinder.

influences of dry and wet modes on dynamic response in typical states are studied, respectively. Further, considering the water load excitation caused by single wave and repeated waves, respectively, the influence of different wave loads on the dynamic response characteristics of the structure is investigated, which can provide a significative reference for the development of the hydroelastic mechanics of amphibious aircraft.

\section{Mathematical Models}

2.1. Wet Modal Analysis. Assuming that water is an isotropic, incompressible ideal fluid, the additional elastic force and additional damping force of water on the structure are ignored; equilibrium equation of normal modes in matrix form of the underwater structure is described as [13]

$$
\left(M+M_{a}\right) \cdot \ddot{x}+C \cdot \dot{x}+K \cdot x=0 .
$$

In the formula, $M$ is the mass matrix, $M_{a}$ is the additional mass matrix generated by the water on the structure, $C$ is the damping matrix, $K$ is the stiffness matrix, and $x$ is the displacement vector.

For underwater structures, the substance of additional mass is the hydrodynamic force per unit of acceleration, which sorbs to the surface of object, and the phase of the force is consistent with the acceleration. This is equivalent to add an additional mass on the surface of the original object, so as to realize the interaction of incompressible fluid to the structure modal characteristics.

Based on the theoretic assumptions of linear elasticity, small deformation, and continuity, the macro large-scale structure is divided into a series of discrete, finite smallscale microelements, and the mechanical features of the whole field are approximately described by the motion of multiple microelements.

When calculating the additional mass produced by surrounding water on a structural microelement $[14,15]$, because water is considered as inviscid, irrotational, and incompressible, the Laplace equation of the flow field can be described as:

$$
\nabla^{2} \kappa=0
$$

In the formula, $\kappa$ is potential function. 
TABLE 1: Natural frequencies of the cylinder.

\begin{tabular}{lccccr}
\hline Mode & Computation Hz & $\begin{array}{c}\text { Dry modal } \\
\text { Test [26] Hz }\end{array}$ & Error & Computation Hz & $\begin{array}{r}\text { Wet modal } \\
\text { Test [26] Hz }\end{array}$ \\
\hline 1 & 196.10 & 194.00 & $1.08 \%$ & 99.15 & 96.00 \\
2 & 202.75 & 198.00 & $2.40 \%$ & 113.57 & $3.28 \%$ \\
3 & 348.39 & 336.00 & $3.69 \%$ & 213.34 & $6.14 \%$ \\
4 & 389.21 & 387.00 & $0.57 \%$ & 221.50 & $7.21 \%$ \\
5 & 409.59 & 403.00 & $1.64 \%$ & 250.73 & 3.00 \\
6 & 570.62 & 565.00 & $0.99 \%$ & 354.04 & 214.00 \\
\hline
\end{tabular}

According to the continuity equation and energy equation of fluid mechanics, the Laplace equation is solved by Helmholtz boundary integral method. The velocity potential at any location on the fluid boundary can be obtained; that is

$$
\dot{x}_{i}=\sum_{i} \int_{s_{i}} \frac{\sigma_{i} e_{i j}}{\left|r_{i}-r_{j}\right|^{2}} \mathrm{~d} s_{i} \text {. }
$$

In the formula, $\dot{\mathrm{x}}_{i}$ is the velocity vector at the node $r_{i}, \sigma_{i}$ is the velocity vector at node $i, e_{i j}$ is the unit vector from point $i$ to point $j$, and $s_{i}$ is the area of the structural surface microelement.

The displacement field of fluid can be described by potential function:

$$
x=\nabla \kappa
$$

By substituting the displacement potential function into the hydromechanical motion equation and integrating,

$$
p=-\rho \ddot{\kappa}
$$

By substituting formula (4) and formula (5) into formula (3), the pressure field can be obtained:

$$
p_{i}=\sum_{i} \int_{s_{i}} \frac{\rho \dot{\sigma}_{i} e_{i j}}{\left|r_{i}-r_{j}\right|} \mathrm{d} s_{i} .
$$

In the formula, $p_{i}$ is the pressure generated by fluid on the structural microelement, and $\rho$ is the fluid density.

Integral Equations (3) and (6), respectively, to obtain as

$$
\begin{aligned}
\dot{x} & =D \cdot \sigma, \\
F & =E \cdot \dot{\sigma} .
\end{aligned}
$$

In the formula, $F$ is the force of the microelement and $D$ and $E$ are the coefficient matrices obtained by numerical integration.

According to the relationship among force, mass, and acceleration, $F$ is

$$
F=M_{a} \cdot \ddot{x}
$$

Substituting Equation (7) and Equation (8) into Equa- tion (9), the virtual mass matrix can be transformed into,

$$
M_{a}=D^{-1} \cdot E
$$

In summary, the natural modes of the underwater structures can be obtained by solving the equilibrium equation. For linear and small disturbance systems, Equation (1) has a solution in the form of simple harmonic function after the additional mass is calculated, and the mechanical problem can be converted into a mathematical eigenvalue analysis. When the eigenvalues and eigenvectors of the motion equation are solved, the natural modal characteristics of the underwater structures can be achieved.

2.2. Hydrodynamic Simulation. For a moving object in water, there are many factors that affect its unsteady hydrodynamic force, including geometry, structural flexibility, moving speed, and wave speed. At present, large numbers of unsteady hydrodynamic researches have been carried out in the field of marine engineering design, and unsteady hydrodynamic calculational methods with medium and low Froude number have been basically formed [16-20].

However, the taxiing speed of amphibious aircraft on the water surface is so high that the Froude number is too large, which belongs to the range of high or ultrahigh Froude number. The unsteady hydrodynamic characteristics are more complex than that of shipping and have exceeded the capabilities of the existing unsteady hydrodynamic numerical theory.

Previous experience has shown that the effect of structural transient deformation to water loads can be ignored in the hydrodynamic design of seaplanes, and the satisfactory results can be obtained in engineering [21]. Therefore, in the absence of a more accurate method, we can refer to the loose coupling theory and adopt the step-by-step solvable idea that is impose the engineering method to calculate the water loads under various wave conditions of the rigid amphibious aircraft in the first instance and then employ the water loads as the hydraulic impact to analyze the dynamic response characteristics of the elastic amphibious aircraft [22].

In the solution, the dynamic effect of the aircraft when it encounters single wave is considered first. The active duration of single wave generally cannot exceed $2 L / V_{b}$ ( $L$ is the wavelength, and $V_{b}$ is the wave velocity), and a certain time range can be selected according to the engineering experience. Many measured results show that the total duration 
of structural loads under water impact is about $0.20 \sim 0.50$ seconds, and the percentage of increase period of pulse forces is between $30 \%$ and $50 \%$ [23].

Furthermore, the engineering design pays more attention to the response characteristics of aircraft when it encounters repeated waves, because it is a high probability that the aircraft encounter repeated waves during the process of taxiing on rough water. In the circumstances, the aircraft may produce a definite frequency of oscillation motion under the continuous excitation of repeated waves.

For the calculation of oscillation frequency, the combination of aircraft velocity and wave velocity should be considered. Among them, the velocity of wave is generally defined as [24]:

$$
V_{b}=\sqrt{\frac{g L}{2 \pi}}
$$

where $g$ is the acceleration of gravity and $L$ is the length of repeated waves. In a general way, a series of wavelengths can be determined according to the ratio range of $L / h$ by given the wave height $h$. The reliable statistics show that $L /$ $h$ is approximate to $8 \sim 40$, for instance.

According to the wavelength, the oscillation frequency when the aircraft encounters repeated waves is expressed as:

$$
f=\frac{V+V_{b}}{L}
$$

where $V$ is the water-taxiing speed of the aircraft.

In the dynamic response analysis of amphibious aircraft induced by water-taxiing, calculations must be made for the range of wave lengths and at least three repeated waves; the oscillation frequency generated by the combination of the aircraft velocity and wave velocity should be consistent with the first $5 \sim 6$ modal frequencies of the aircraft structure. These are generally considered to be the most severe predicament that may be encountered.

2.3. Aerodynamic Simulation. In engineering design, Doublet Lattice Method is widely used to calculate the unsteady aerodynamic force of lift surfaces, but it can only obtain the aerodynamic force in frequency domain. In the transient dynamic response analysis, aerodynamic force should be converted to the form of time domain. The MinimumState Approximation Formula can be selected to time domain fitting of the unsteady aerodynamic force [25].

Firstly, the generalized aerodynamic force in frequency domain can be extended to Laplace domain:

$$
Q_{A}=A_{0}+A_{1} p+A_{2} p^{2}+D \cdot(I p-R)^{-1} \cdot E p,
$$

where $p=s b / V$ is the dimensionless Laplace variable; $A_{0}$, $A_{1}, A_{2}, D$, and $E$ are the polynomial coefficient matrix; $I$ is the unit matrix; $R$ is the aerodynamic lag coefficient matrix; and $n$ is the number of lag terms.

Based on the small disturbance assumption, the lift surface motion can be regarded as harmonic oscillation. If $p=$ $i k$, the generalized aerodynamic force is expressed as:

$$
Q_{A}(i k)=F_{h}(k)+i G_{h}(k)
$$

$$
\begin{aligned}
& F_{h}(k)=A_{0}-k^{2} A_{2}+k^{2} D \cdot\left(k^{2} I+R^{2}\right)^{-1} E, \\
& G_{h}(k)=k A_{1}-k D \cdot\left(k^{2} I+R^{2}\right)^{-1} E R,
\end{aligned}
$$$$
\text { where } k=\omega b / V \text {. }
$$

The generalized aerodynamic force in time domain is possible to obtain by solving multidimensional equations according to the nonlinear least-square solution and Laplace inverse transformation:

$$
Q_{A}(t)=q_{\infty}\left(\bar{A}_{0} \xi+\frac{b}{V} \bar{A}_{1} \dot{\xi}+\frac{b^{2}}{V^{2}} \bar{A}_{2} \ddot{\xi}+D u\right)
$$

$\dot{u}(t)=A_{c} \xi+(V / b) R \cdot u(t)$,

where $A_{C}$ is the aerodynamic coefficient matrix and $u$ is the additional state vector.

2.4. Motion Equation of Elastic Aircraft. After calculating the wet modal characteristics, hydrodynamic force, and aerodynamic force, dynamic response equation for damping motion under a transient excitation is described as

$$
\left(M+M_{a}\right) \cdot \ddot{x}+C \cdot \dot{x}+K \cdot x=P_{h}(t)+Q_{A}(t) .
$$

In the formula, $P_{H}$ is the transient hydrodynamic excitation in the time domain and is a function of time.

In order to decouple the equation, a transformation is needed between the physical displacement and the modal coordinate:

$$
x(t)=\phi \cdot \xi(t)
$$

In the equation, $\phi$ is mode shape, and $\xi$ is modal coordinate.

And Equation (16) is converted to as follows:

$\phi^{T}\left(M+M_{a}\right) \phi \cdot \ddot{\xi}(t)+\phi^{T} C \phi \cdot \dot{\xi}(t)+\phi^{T} K \phi \cdot \xi(t)=\phi^{T}\left(P_{h}(t)+Q_{A}(t)\right)$.

According to the orthogonal characteristic of mode shapes, the generalized equation can be transformed into a series of uncoupled single degree of freedom system. Whereafter the central difference method is used to solve the uncoupled equations in time axis, and the transient response course can be obtained by superimposing the modal responses of each order.

\section{Verification of Wet Modal Analysis Method}

To verify the validity of the Virtual Mass Theory, wet modal characteristics of an unconstrained cylinder submerged in water are analyzed. The cylinder has the following dimensions: length $h=1.284 \mathrm{~m}$, diameter $D=0.357 \mathrm{~m}$, and wall and end cap thickness $t=0.003 \mathrm{~m}$, while the material properties are Poisson's ratio $v=0.29$, density $\rho=7750 \mathrm{~kg} / \mathrm{m}^{3}$, and Young's modulus $E=207 \mathrm{GPa}$. 


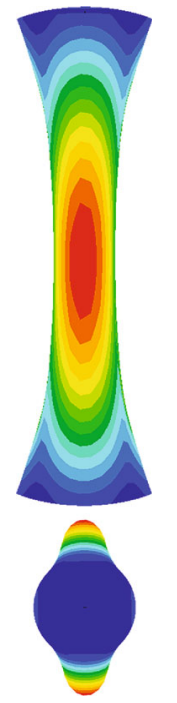

(a) $f=99.15 \mathrm{~Hz}$

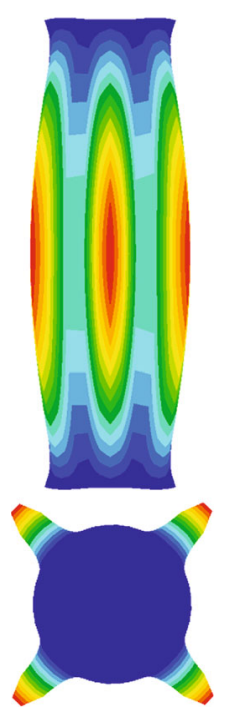

(b) $f=213.34 \mathrm{~Hz}$

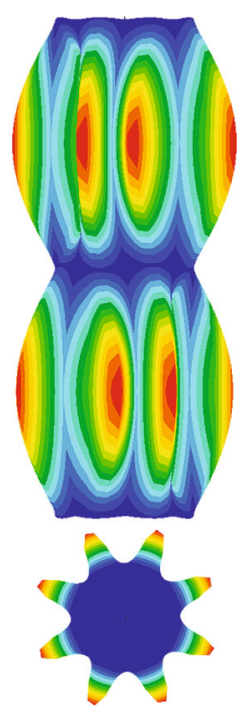

(c) $f=250.73 \mathrm{~Hz}$

FIgURE 2: Typical wet mode shapes of the cylinder.

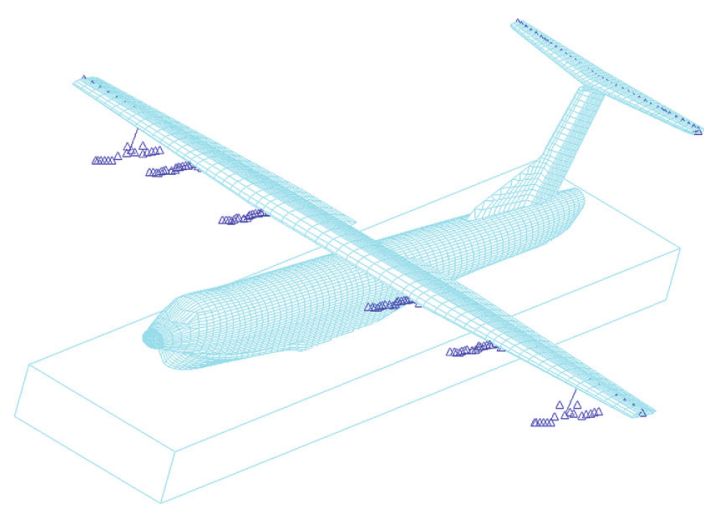

Figure 3: Wet model of large amphibious aircraft.

Finite element model of the cylinder is established according to the structure layout as shown in Figure 1. Then, the modal characteristics of the cylinder in dry and wet conditions are calculated, respectively. Results show that compared with the dry modes, the wet natural frequencies of the cylinder decrease greatly after considering the effect of water added mass. It follows that for underwater structures, the influence of water added motion on structural modes cannot be disregarded.

The comparisons of cylinder modal analysis obtained by the present approach and the experimental values given by Price et al. [26] are shown in Table 1. It can be seen that the calculated values of the listed frequencies are in good consistencies with the experimental values, whether in dry modal or wet modal. The maximal differences of dry and wet natural frequencies between analytical and experimental are 3.69\% and $7.21 \%$, respectively, for the 6 modes of major concern.

The typical wet mode shapes are shown in Figure 2. It is thus clear that the good symmetry of wet mode shapes of the cylinders is demonstrated, which looks very artistic, and the circumferential wave numbers and axial wave numbers of the mode shapes are well agreement with the test results.
Taking the above conclusions into account, the analysis in this work is both accurate and reliable since the analytical results are basically in agreement with the experimental results; it can be applied to the wet modal analysis of amphibians as the fuselage is similar to a roughly cylindrical thing.

\section{Modeling and Analysis}

4.1. Wet Modal Analysis. In this work, the large amphibious aircraft has a configuration of single-hull fuselage, high-wing with large aspect ratio, and T-tail; four turboprop engines are mounted at the leading edge of the wing, and a stabilizing float is installed under the each side of outer wings. The wing span is $38.80 \mathrm{~m}$, the length and the height of the aircraft are $36.94 \mathrm{~m}$ and $12.10 \mathrm{~m}$, and the maximum takingoff weight is $60000 \mathrm{~kg}$.

Base on the configuration and structural sizing of the large amphibious aircraft, the structural dynamic model is created using the elements of $1 \mathrm{D}$ beam, $2 \mathrm{D}$ shell, and $0 \mathrm{D}$ concentrated mass as shown in Figure 3. During the modeling, stiffness distribution of the load-carrying structures of the aircraft is sufficiently simulated. The skin and web are represented by shell elements; the stringer, spar, and keel are represented by beam elements, and all the components are connected by flexible elements. In order to improve the calculational efficiency, the engines, floats, landing gears, control surfaces, etc., are aptly simplified as mass elements, and their own stiffness are ignored in the model. In addition, according to the mass distribution of structures, equipments, payloads, etc., multiple centralized mass elements are used to simulate the mass distribution of the whole aircraft. In addition, the structural model is verified by the Ground Vibration Test of the full-scale aircraft with good correlation.

In the calculation, the height of the waterline can be obtained according to loading status and water-taxiing parameters of the aircraft; the hull below the waterline is in 
TABLE 2: Computation of modal frequencies.

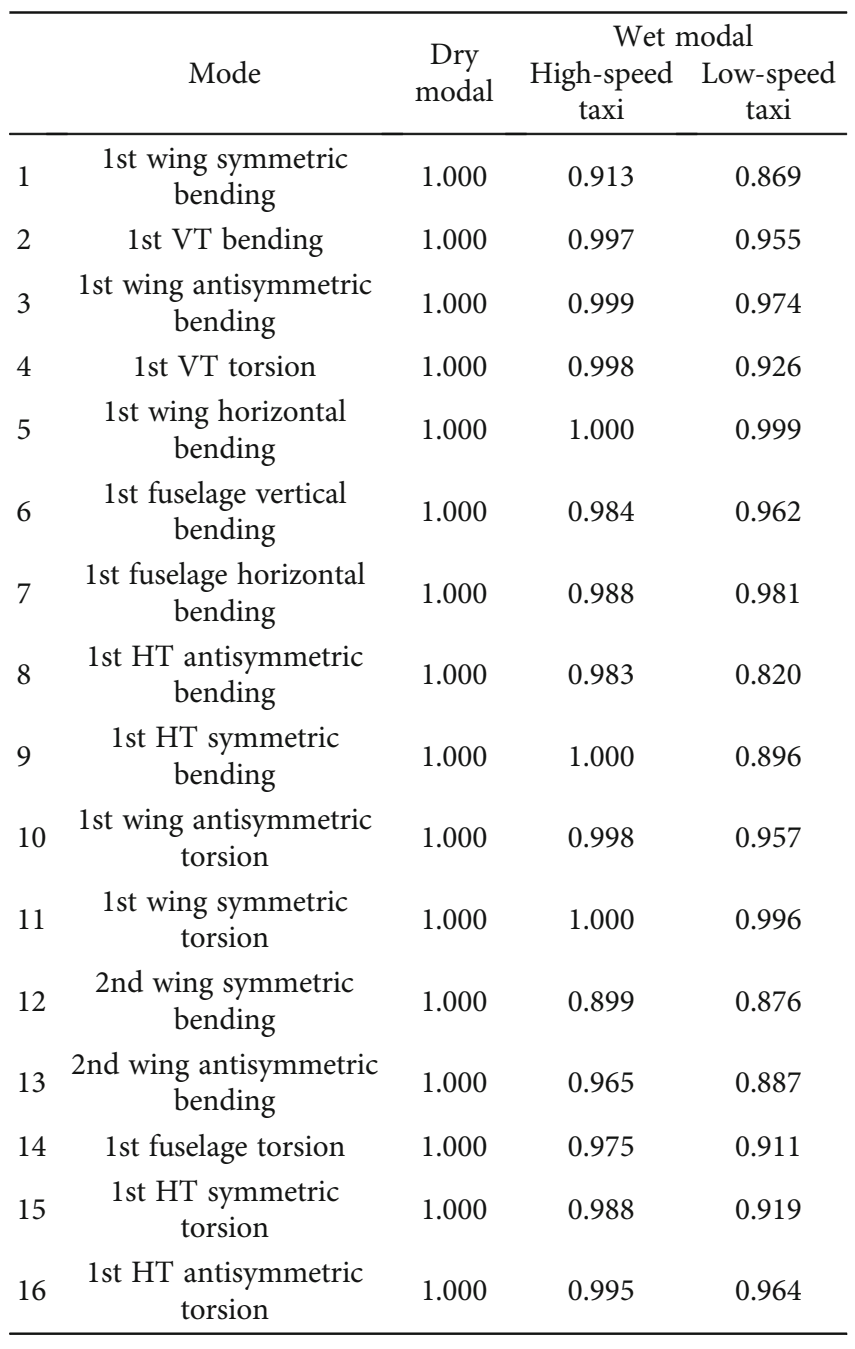

contact with water as shown in Figure 3. In this way, using the parameters of underwater hull, we can determine the additional mass produced by the interaction between water and hull, and then, the wet modes of the aircraft can be solved.

Table 2 gives the dry and wet natural frequencies by calculation in typical states of large amphibious aircraft. In order to facilitate comparison, the wet frequencies are normalized of dry modal. The results show that when in different taxiing speed on water surface, the height of the waterline is also different resulting in different additional mass of the hull, and the wet frequencies are different likewise. Compared with the dry modal of the aircraft, the wet frequencies decrease in varying degrees, but both of the mode shapes are basically similar.

Typical wet mode shapes of large amphibious aircraft are shown in Figures 4 and 5.

4.2. Comparison of Response between Wet and Dry Modes. In the past, the mode superposition method based on the dry modes was mainly used for the dynamic response of amphibious aircraft when it was moving on the water surface, and the effect of unsteady aerodynamic force on the

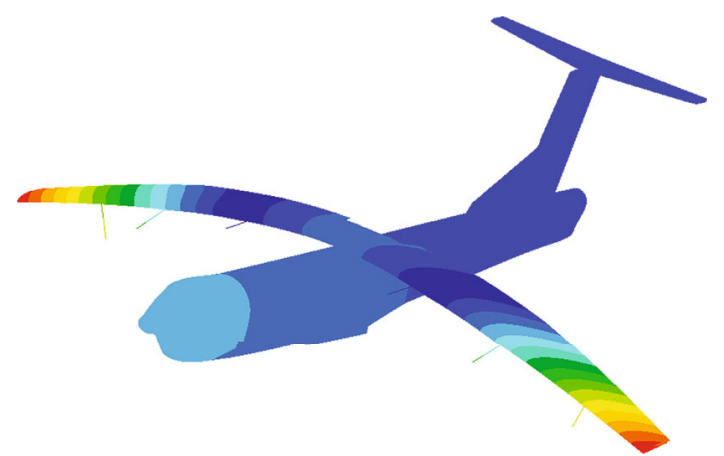

FIGURE 4: Wet mode shape of 1st wing symmetric bending.

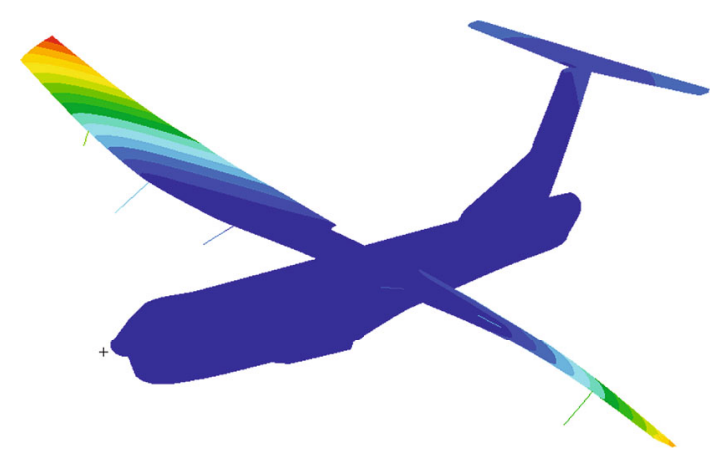

FIGURE 5: Wet mode shape of 1st wing antisymmetric bending.

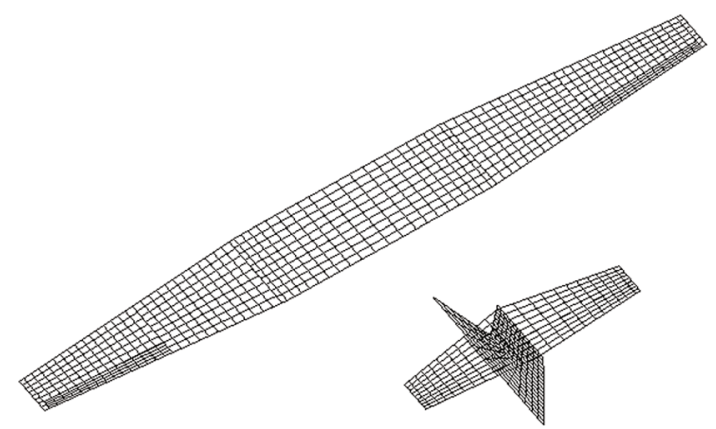

FIGURE 6: The configuration of lifting surfaces.

lifting surfaces was usually ignored; these were all inconsistent with the actual situation.

In order to simulate the dynamic response more accurately, the unsteady aerodynamic modeling of large amphibious aircraft is carried out in this work, the configuration of lifting surfaces as shown in Figure 6. The aerodynamic force in frequency domain on the lifting surfaces is calculated by Doublet Lattice Method.

According to the simplified time history of water load under single wave as shown in Figure 7, the hydrodynamic load factor of the aircraft by empirical formula [1] is $n_{z}=$ $3.75 \mathrm{~g}$, the structural damping coefficient obtained from Ground Vibration Test is $g=0.02$, and then the dry and wet modes are taken to analyze the transient response of large amphibious aircraft in typical state, respectively; the results are as follows. 


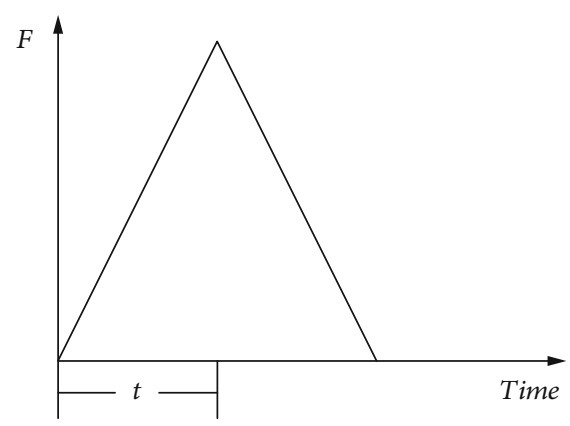

Figure 7: The simplified time history of water load $(t=0.06$ $\sim 0.25 \mathrm{~s})$.

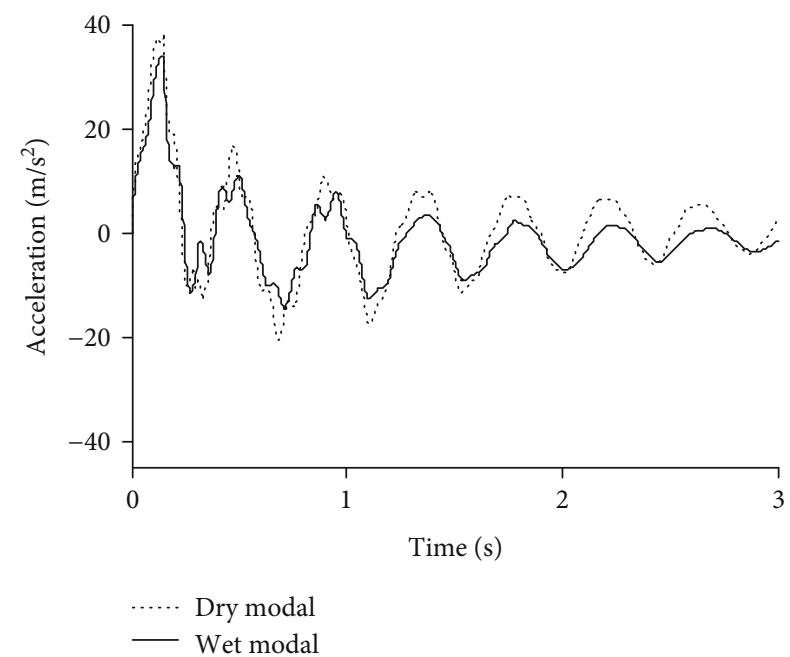

Figure 8: Transient response of acceleration at the center of gravity.

As shown in Figures 8-10, the oscillatory amplitude in different locations of the aircraft changes in varying degrees relative to the center of gravity due to the influence of structural elasticity and especially at the outside of the wing, which is even more than twice that of the center of gravity in local position. It is inevitable that the water load factors of various parts cannot be simply described by the center of gravity overload when considering the airframe elasticity.

Meanwhile, the oscillatory trends of wet and dry modes are basically consistent, but the oscillatory amplitude is different. The peak values of normal acceleration of the center of gravity and wing tip obtained by dry modes are $38.51 \mathrm{~m} / \mathrm{s}^{2}$ and $-80.56 \mathrm{~m} / \mathrm{s}^{2}$, respectively, while those obtained by wet modes are $34.28 \mathrm{~m} / \mathrm{s}^{2}$ and $-66.57 \mathrm{~m} / \mathrm{s}^{2}$, respectively; it is thus clear that the amplitude of wet modal response decreases. This is mainly due to the influence of additional mass in the hull area of the aircraft.

Some existing disquisitive results show that considering the impact of the dynamic response of the water-taxing, the dynamic loads of the aircraft are significantly greater than the static loads. Supplementary design changes of the structure must be implemented according to the dynamic water loads, so as to ensure the structural strength safety [7]. Considering this, the dynamic response of wet modes should be used in the analysis of the hydrodynamic charac-

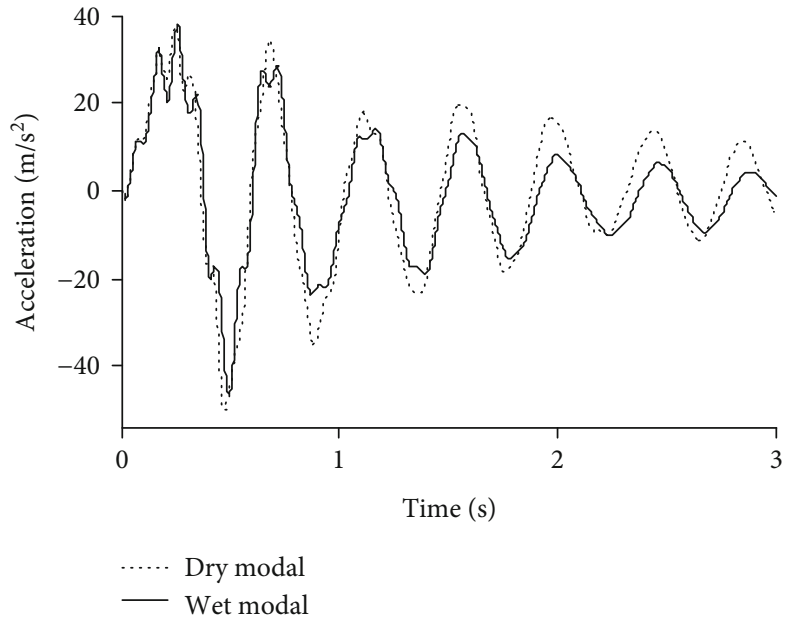

FIgURE 9: Transient response of acceleration at mid wing.

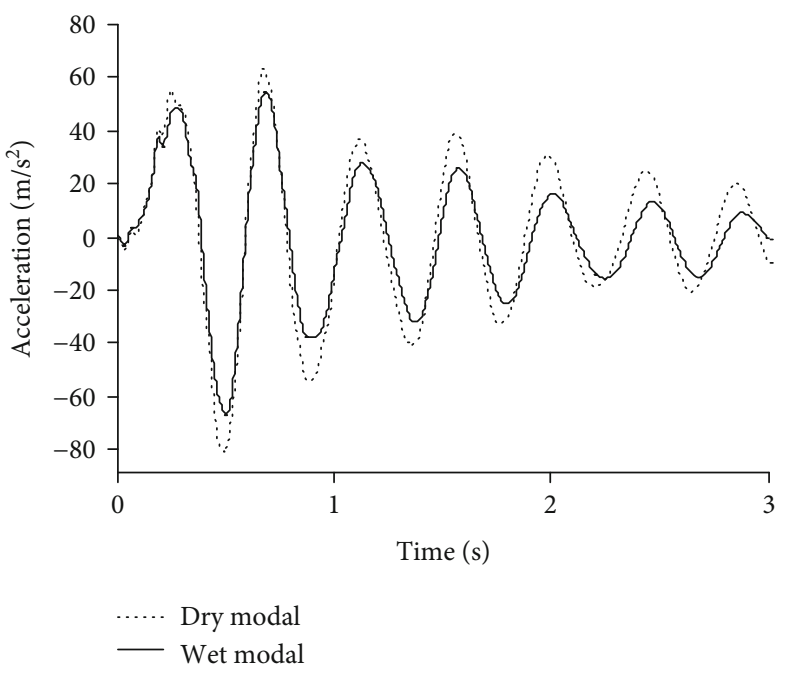

FIGURE 10: Transient response of acceleration at wing tip.

teristics of large amphibious aircraft. Otherwise, the dynamic load amplitude of the aircraft structure obtained from the dry modal analysis will be inaccurate, which will likely lead to the conservative design of the structural strength and unnecessary structural weight gain.

4.3. Dynamic Response Analysis of Water-Taxiing. Furthermore, according to the models shown in Figures 3 and 6, the damping coefficient of the structure is given as $g=0.02$ similarly; the simplified time history of water loads including single wave and repeat waves is, respectively, used to solve the dynamic response at taxiing on rough water surface of large amphibious aircraft by the wet mode superposition method.

Under the action of single wave, the acceleration response courses of typical parts of fuselage and wing are shown by the solid line in Figures $8-10$. It can be seen that under the influence of water load generated by single wave, the acceleration response of the structure will increase rapidly to the peak value and then gradually attenuate under 


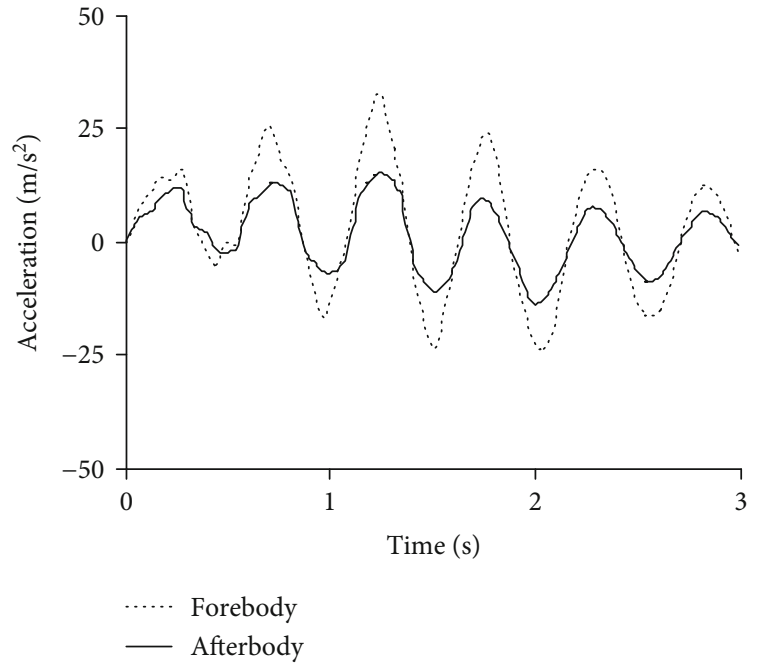

FIgURE 11: Transient response of acceleration at typical sections of fuselage under repeated waves.

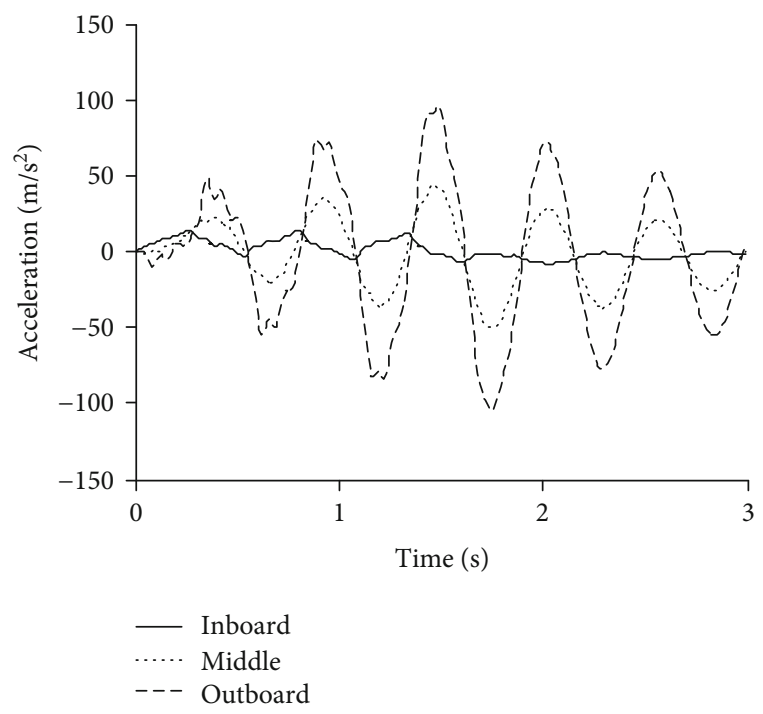

Figure 12: Transient response of acceleration at typical sections of wing under repeated waves.

the effect of structural damping. In this situation, the first response peak is usually considered in structural strength design.

For repeated waves, first five elastic modes of the aircraft are mainly 1st wing symmetric bending, 1st VT bending, 1st wing antisymmetric bending, 1st VT torsion, and 1st wing horizontal bending. Because the direction of the water load is vertical, the most affected elastic modes are 1st wing symmetric bending and 1st wing antisymmetric bending. These frequencies are taken as the oscillation frequencies generated by the combination of aircraft velocity and wave velocity.

Considering the periodic action of three repeated waves, under the influence of the oscillation frequency equal to the wing modal frequencies, the transient response courses of acceleration at each part of the aircraft under the action of water load generated by repeated waves are solved. The typical results are shown in Figures 11-14, respectively.

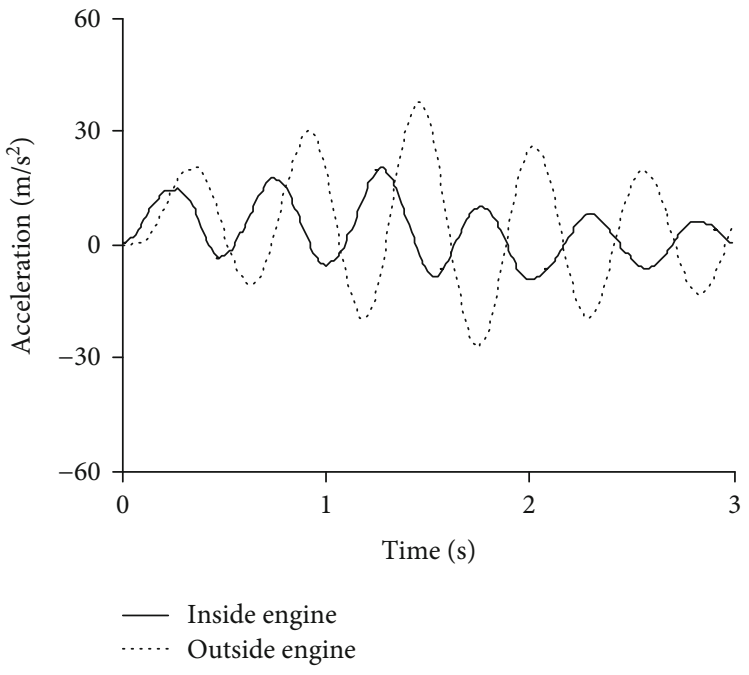

FIGURE 13: Transient response of acceleration at center of gravity of engines under repeated waves.

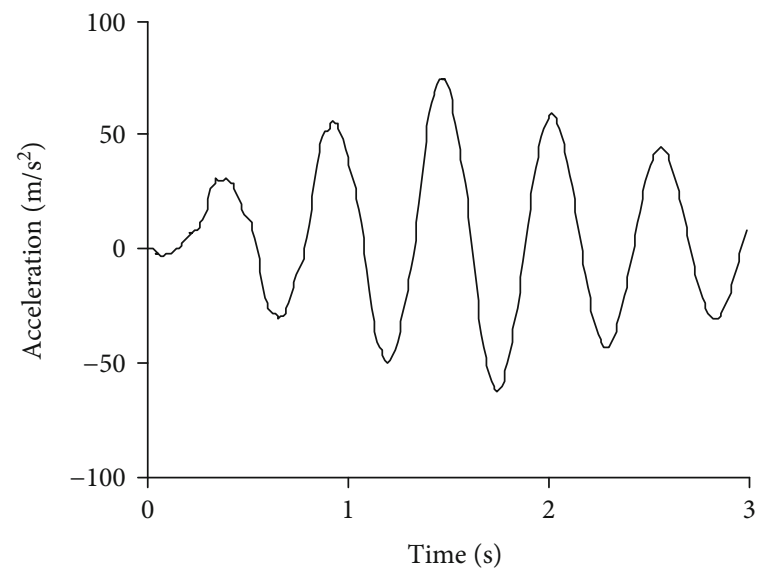

FIgURE 14: Transient response of acceleration at center of gravity of float under repeated waves.

It can be seen that under the action of typical oscillation frequency and the influence of repeated waves excitations, the acceleration response of the structure will increase rapidly, and the peak values of the response show evident divergent trend. That is, the peak values of the response will continue to increase within the time range of excitation and gradually attenuate under the effect of structural damping beyond the excitation. The main reason is that the oscillation frequency of the aircraft under the action of repeated waves is consistent with the elastic modal frequency, which induces the structural resonance phenomenon and makes the structure produce divergent dynamic response because of the limited structure damping. This situation is more severe for structural design; the maximum response peaks including positive and negative must be fully considered usually.

Moreover, the results show that the symmetrical bending mode of the wing is easier to be excited under the dynamic excitation of repeated wave loads, and the distribution of the mode shape has a direct impact on the response amplitude of different regions. As shown in Figure 4, the modal 
magnitude of the front fuselage and outer wing is larger than that of the rear fuselage and the inner wing, respectively, which makes the acceleration response of the front fuselage and the outer wing correspondingly larger, as shown in Figures 11 and 12. Based on this, it is more advantageous for structural strength design to place some important equipments at or close to the nodal line location of wing bending mode.

In addition, for large external stores such as wing cantilevered engines and wing suspended floats, inertial forces are mainly used for structural strength design of the installations. It should be paid especial attention that the inertial impact loads on external stores will continue to increase under repeated wave excitation as shown in Figures 13 and 14, which is likely to become the critical condition of strength design.

\section{Conclusions and Prospects}

In the past, the dynamics research of aircraft in water mainly focuses on the water landing or ditching and also ignores the influence of structural elasticity to water loads. In addition, when investigating the structural modal and dynamic response characteristics of seaplane, it also ignores the influence of water on the structure and usually uses dry modes rather than wet modes, which are all inconsistent with the actual situation.

In order to solve the structural dynamic problems of large amphibious aircraft on the water surface in this work, the whole aircraft structural dynamic model is established according to the stiffness and mass distribution, the Virtual Mass Theory is adopted to analyze the wet modes refer to the waterline of the hull, the Minimum-State Approximation Formula is used to fit the unsteady aerodynamic force in time domain, the loose coupling method is utilized to carry out the numerical simulation of the hydrodynamic elastic response, and the influences of dry and wet modes, single wave, and repeated waves on the dynamic response characteristics in typical states have been investigated severally; the results show that:

(1) Considering the additional mass caused by water for the large amphibious aircraft, the wet frequencies decrease in varying degrees compared with dry frequencies, when as both of the mode shapes are relatively likeness

(2) Take into account the influence of structural elasticity, the oscillatory amplitude of different location in water-taxiing is variable respect to the center of gravity, and the water load factor of each section cannot be described by the load factor at center of gravity

(3) The loose coupling method is employed, and the transient response analysis results obtained by wet modes are different from those obtained by dry modes; the structural response trends are basically consistent, but the oscillatory amplitude of wet modes decreases to a certain extent
(4) When the aircraft encounters a single wave, the structural oscillatory amplitude will increase rapidly to the peak value under the influence of water load excitation and then gradually attenuate because of the effect of structural damping. Structural strength design only needs to pay attention to the first response peak

(5) The response amplitude will increase rapidly when the aircraft encounters repeated waves, and the responses are divergent by the repeated load excitation at a certain oscillation frequency. This situation is more severe for structural design, and the maximal response peak must be adequately considered

The disquisitive results are of great significance for the development of the theory of hydrodynamics on amphibious aircraft. In the future, it is necessary to further investigate the dynamic simulation modeling and unsteady hydrodynamic characteristics of large amphibious aircraft in the specific service environment and establish an integrated technical theory to analyze the motion and deformation of the water structure as a general holonomic system, so as to provide some available technical support for the hydroelastic dynamic design of hydroplane and amphibious aircraft.

\section{Data Availability}

All data, models, and code generated or used during the study appear in the submitted article.

\section{Conflicts of Interest}

The authors declare that there is no conflict of interest regarding the publication of this article.

\section{Acknowledgments}

This paper is a part of a research project supported by the Department of R\&D Centre and S\&T Committee, AVIC General Huanan Aircraft Industry Limited Company. Thanks are due to Dr. Linyin Luo from S\&T Committee of AVIC General Huanan Aircraft Industry Limited Company for his technical support on this article.

\section{References}

[1] General Administration of Civil Aviation of China, CCAR-25R4, China Civil Aviation Regulations, Part 25 Airworthiness Standards: Transport Category Airplanes, General Administration of Civil Aviation of China, Beijing, 2011.

[2] Federal Aviation Administration, FAR-25 Airworthiness Standards: Transport Category Airplanes, Federal Aviation Administration, Washington, 2014.

[3] Q. Qu, M. Hu, H. Guo, P. Liu, and R. K. Agarwal, "Study of ditching characteristics of transport aircraft by global moving mesh method," Journal of Aircraft, vol. 52, no. 5, pp. 15501558, 2015.

[4] Z. Yi, S. Yang, and L. Luo, "The structural response of amphibian landing onto wave," Journal of Machine Design, vol. 32, no. 9, pp. 96-100, 2015. 
[5] L. T. Chu, B. Wu, and M. Z. Wang, "Theoretical calculation and experiment on impact loads of landing of wing-inground on water surface," Acta Aeronautica et Astronautica Sinica, vol. 37, no. 12, pp. 3698-3705, 2016.

[6] S. A. Fazelzadeh and H. S. Hoseini, "Nonlinear flight dynamics of a flexible aircraft subjected to aeroelastic and gust loads," Journal of Aerospace Engineering, vol. 25, no. 1, pp. 51-63, 2012.

[7] L. Jihang, Z. Yi, and R. Yang, "Dynamic response characteristics of large amphibious aircraft," Aeronautical Manufacturing Technique, vol. 63, no. 20, pp. 64-69, 2020.

[8] E. N. Abdulwahab and C. Hongquan, "Aircraft response to atmospheric turbulence at various types of the input excitation," Space Research Journal, vol. 1, no. 1, pp. 17-28, 2008.

[9] E. H. van Brummelen, "Added mass effects of compressible and incompressible flows in fluid-structure interaction," Journal of Applied Mechanics, vol. 76, no. 2, article 021206, 2009.

[10] O. De La Torre, X. Escaler, E. Egusquiza, and M. Farhat, "Numerical and experimental study of a nearby solid boundary and partial submergence effects on hydrofoil added mass," Computers and Fluids, vol. 91, no. 6, pp. 1-9, 2014.

[11] Z. Wenpeng and Z. Zhi, "3D analysis method for added mass matrix about free vibration of structure in water," Chinese Journal of Ship Research, vol. 6, no. 4, pp. 13-18, 2011.

[12] L. Xinmin and Z. Jinghui, "Approximate evaluation and analysis for natural frequency of circular cylinder shell in water," Chinese Journal of Applied Mechanics, vol. 25, no. 2, pp. 326332, 2008.

[13] L. Monterrubio and P. Krysl, "Natural frequencies of submerged structures using an efficient calculation of the added mass matrix in the boundary element method," Journal of Vibration and Acoustics, vol. 141, no. 2, pp. 1-9, 2019.

[14] M. Donald and M. Lean, MD Nastran Dynamic Analysis User's Guide, MSC Software Corporation, Los Angeles, 2010

[15] C. Ma, Z. Xinong, and Y. Luo, "Dynamic analysis on a fluidstructure coupling system with variable mass in a rectangular tank," Journal of Xi'an Jiaotong University, vol. 48, no. 7, pp. 109-116, 2014.

[16] R. Bishop and W. G. Price, "An introduction to ship hydroelasticity," Journal of Sound \& Vibration, vol. 87, no. 3, pp. 391-407, 1983.

[17] I. Senjanovic, S. Malenica, and S. Tomasevic, "Investigation of ship hydroelasticity," Ocean Engineering, vol. 35, no. 5-6, pp. 523-535, 2008.

[18] Y. Kim, I. G. Ahn, and S. G. Park, "Extraction of the mode shapes of a segmented ship model with a hydroelastic response," International journal of naval architecture and ocean engineering, vol. 7, no. 6, pp. 979-994, 2015.

[19] Z. Y. Chen, H. B. Gui, and H. L. Ren, "Direct calculation method for nonlinear design loads of warship based on hydroelasticity theory," Journal of Harbin Engineering University, vol. 38, no. 1, pp. 37-42, 2017.

[20] Y. S. WU, M. S. ZOU, J. DING et al., "Three-dimensional hydroelasticity theory of ships in waves and ocean-acoustic environment with applications," SCIENTIA SINICA Physica, Mechanica \& Astronomica, vol. 48, no. 9, p. 094602, 2018.

[21] Aircraft Design Manual Compilatory Committee, Aircraft Design Manual: The Ninth Volume, Loads, Strength and Stiffness, Aviation Industry Press, Beijing, 2001.

[22] Beriev Design Bureau, Beriev Be-200ES-E Special Conditions, Taganrog: TANTK Beriev Aircraft Company, 2007.
[23] A. A. Goryanov, Volume II Aircraft Designer's Manual: Hydrodynamics of Seaplane, Central Institute of Aerodynamic Dynamics, Moscow, 1938.

[24] R. Bhattacharyya, Dynamic of Marine Vehicles, A WileyInterscience Publication, New York, 1978.

[25] M. J. Henshawa, K. J. Badcock, and G. A. Vio, "Non-linear aeroelastic prediction for aircraft applications," Progress in Aerospace Sciences, vol. 43, no. 4-6, pp. 65-137, 2007.

[26] W. G. Price, R. Randall, and P. Temarel, Fluid-Structure Interaction of Submerged Shells, Naval Architecture and Offshore Engineering Conference, Surrey, UK, 1988. 